VOL. 60 (1999) [331-334]

\title{
A COMPLETE CLASSIFICATION OF FINITE HOMOGENEOUS GROUPS
}

\author{
Cai Heng Li
}

In this short note, we obtain a complete classification of finite homogeneous groups.

A group $G$ is called a homogeneous group if each isomorphism between any two isomorphic subgroups of $G$ extends to an automorphism of $G$. A classification of finite soluble homogeneous groups was published in [3]. The purpose of this note is to give a complete classification of arbitrary finite homogeneous groups. A group is called a homocyclic group if it is a direct product of cyclic subgroups of the same order. For two groups $G$ and $H$, denote by $G \rtimes H$ a semidirect product of $G$ by $H$. We use Q(64) to denote a Sylow 2-subgroup of PSU $(3,16)$, which is a Suzuki 2-group of order 64 . The main result of this paper is the following theorem.

MarN Theorem. A finite group $G$ is homogeneous if and only if $G=U \times V$ such that $(|U|,|V|)=1, U$ is Abelian with all Sylow subgroups homocyclic, and either $V=1$, or $V$ is one of the following:

(1) $W \rtimes \mathbb{Z}_{2^{n}}$, where $W$ is Abelian of odd order with all Sylow subgroups homocyclic, and $\mathbb{Z}_{2^{n}}$ inverses all elements of $W$;

(2) $\mathrm{Q}_{8}$ and $\mathrm{Q}(64)$;

(3) $\mathrm{A}_{4}, \mathrm{Q}_{8} \rtimes \mathbb{Z}_{3}, \mathbb{Z}_{3}^{2} \rtimes \mathrm{Q}_{8}$ and $\mathrm{Q}(64) \rtimes \mathbb{Z}_{3}$;

(4) $\mathrm{L}_{2}(5), \mathrm{L}_{2}(7), \mathrm{SL}_{2}(5)$ and $\mathrm{SL}_{2}(7)$.

One of the motivations for the study of homogeneous groups comes from model theory, that is, a complete first-order theory that admits quantifier elimination has a homogeneous model. In particular, a finite structure is homogeneous if and only if it admits quantifier elimination, see $[2,3]$. This would be the principal motivation for the work of Cherlin and Felgner in $[2,3]$.

However, the motivation for the present work comes from graph theory, namely a problem about isomorphisms of Cayley graphs, see $[5,8]$ for references. In the study of this problem, we need to know the homogeneity of some groups, see [5]. Although it was claimed in [3] that a classification of finite homogeneous groups had been obtained,

Received July 29, 1999

Partially supported by an ARC small grant.

Copyright Clearance Centre, Inc. Serial-fee code: $0004-9727 / 99 \$ A 2.00+0.00$. 
such a classification has never been published so far. Because of the demand for such a classification and the briefness of the argument, we in this short note give a complete classification of finite homogeneous groups.

Some properties of finite groups similar to homogeneity have been investigated, for example, [4] proved that a group of which all elements of the same order are conjugate is isomorphic to $\mathrm{S}_{2}$ or $\mathrm{S}_{3} ;[10]$ gave a description for finite groups $G$ such that all elements of the same order are conjugate in $\operatorname{Aut}(G)$; Praeger and the author in $[6,7]$ obtained a description for finite groups $G$ in which any two elements of the same order are conjugate or inverse-conjugate in Aut $(G)$; Stroth [9] classified finite groups in which all isomorphic subgroups are conjugate.

The rest of the note is devoted to giving a short proof of the Main Theorem.

Proof of THE MAIN TheOrem: Assume that $G$ is a finite homogeneous group. If $G$ is soluble, then by [3], $G$ is on the list in the theorem. Thus we assume that $G$ is insoluble. Since $G$ is homogeneous, all elements of the same order are conjugate in Aut $(G)$. Then by [8, Corollary 2.4] and [7, Corollary 1.3(4)], $G=U \times V$ such that $(|U|,|V|)=1, U$ is Abelian of odd order with all Sylow subgroups homocyclic, and $V$ is one of $\mathrm{L}_{2}(5), \mathrm{L}_{2}(7), \mathrm{L}_{2}(8), \mathrm{L}_{2}(9), \mathrm{L}_{3}(4), \mathrm{SL}_{2}(5), \mathrm{SL}_{2}(7)$ and $\mathrm{SL}_{2}(9)$. Thus we need to prove that none of $\mathrm{L}_{2}(8), \mathrm{L}_{2}(9), \mathrm{L}_{3}(4)$ and $\mathrm{SL}_{2}(9)$ is homogeneous.

Assume that $V$ is one of $\mathrm{L}_{2}(8), \mathrm{L}_{2}(9), \mathrm{SL}_{2}(9)$ and $\mathrm{L}_{3}(4)$. Let $K$ be a subgroup of $V$. Suppose that $V$ is homogeneous. Then by the definition, all automorphisms of $K$ extend to automorphisms of $V$. Suppose further that $K$ is elementary Abelian, and let $\Omega$ be the set of minimal generating subsets for $K$. Then it follows since $V$ is homogeneous that $\mathrm{N}_{\mathrm{Aut}(V)}(K)$ is transitive on $\Omega$ and so induces a transitive permutation group $P$ on $\Omega$. Clearly, $P \cong \mathrm{N}_{\mathrm{Aut}(V)}(K) / \mathrm{C}_{\mathrm{Aut}(V)}(K)$.

Suppose first that $V=\mathrm{L}_{2}(8)$ is homogeneous. Then by [1], a Sylow 2-subgroup $K$ of $V$ is isomorphic to $\mathbb{Z}_{2}^{3}$, and $P \cong \mathbf{N}_{\text {Aut }(V)}(K) / \mathbf{C}_{\text {Aut }(V)}(K) \cong \mathbb{Z}_{7} \rtimes \mathbb{Z}_{3}$. Since every 3 non-identity elements of $K$ generate a subgroup isomorphic to $\mathbb{Z}_{2}^{2}$ or $\mathbb{Z}_{2}^{3}$, it easily follows that $|\Omega|=\left(\begin{array}{l}7 \\ 3\end{array}\right)-\left(\begin{array}{l}7 \\ 2\end{array}\right)=14$. Thus $|\Omega|$ does not divide $|P|$, and so $P$ is not transitive on $\Omega$, which is a contradiction. Therefore, $\mathrm{L}_{2}(8)$ is not homogeneous.

Suppose next that $V=\mathrm{L}_{2}(9)$ is homogeneous. By the Atlas [1], a Sylow 3-subgroup $K$ of $V$ is isomorphic to $\mathbb{Z}_{3}^{2}$, and $P \cong \mathbf{N}_{\text {Aut }(V)}(K) / \mathbf{C}_{\text {Aut }(V)}(K)$ is a group of order 16 . Now $K$ has 8 non-identity elements and any two of them generate a subgroup isomorphic to $\mathbb{Z}_{3}$ or $\mathbb{Z}_{3}^{2}$. It follows that $|\Omega|=\left(\begin{array}{l}8 \\ 2\end{array}\right)-\left(\begin{array}{l}8 \\ 1\end{array}\right)=20$. Thus $|\Omega|$ does not divide $|P|$, and so $P$ is not transitive on $\Omega$, which is a contradiction. So $L_{2}(9)$ is not homogeneous. The same argument shows that $\mathrm{SL}_{2}(9)$ is not homogeneous.

Suppose finally that $V=\mathrm{L}_{3}(4)$ is homogeneous. By the Atlas [1], $V$ has a maximal subgroup $M=K \rtimes H$ such that $K \cong \mathbb{Z}_{2}^{4}$ and $H \cong \mathrm{A}_{5}$. We notice that any subset of 4 non-identity elements of $K$ generates a subgroup isomorphic to $\mathbb{Z}_{2}^{2}, \mathbb{Z}_{2}^{3}$ or $\mathbb{Z}_{2}^{4}$. It is easily shown that $|\Omega|=\left(\begin{array}{c}15 \\ 4\end{array}\right)-\left(\begin{array}{c}15 \\ 3\end{array}\right)+\left(\begin{array}{c}15 \\ 2\end{array}\right)$ and is divisible by 29 . On the other hand, 
as $V$ is homogeneous, all minimal generating subsets of $K$ are conjugate in Aut $(V)$, which is a contradiction since Aut $(V)$ is of order coprime to 29. Therefore, $L_{3}(4)$ is not homogeneous.

Conversely, we need to prove that all groups listed in the theorem are homogeneous. By [3, Proposition 8], $U$ is homogeneous, and by [5, Lemma 3.1], if $V$ is homogeneous then $G=U \times V$ is homogeneous. Thus we need to verify that $V$ is homogeneous. By [3, Proposition 8], if $V$ is soluble then $V$ is homogeneous. Hence we only need to show that the groups listed in item (3), namely $\mathrm{L}_{2}(5), \mathrm{L}_{2}(7), \mathrm{SL}_{2}(5)$ and $\mathrm{SL}_{2}(7)$, is homogeneous.

(1) Assume that $V=\mathrm{L}_{2}(p)$, where $p=5$ or 7 .

Let $K, L<V$ be such that $K \cong L$, and let $\sigma$ be an isomorphism from $K$ to $L$. From the information given in the Atlas [1], we easily conclude that all elements of $V$ of the same order are conjugate in $\operatorname{Aut}(V)$. It follows that if $K, L$ are cyclic then $\sigma$ extends to an automorphism of $V$. Thus we suppose that $K$ is not cyclic. Then $K \cong \mathbb{Z}_{2}^{2}, \mathrm{D}_{6}, \mathrm{D}_{8}$, $\mathrm{D}_{10}, \mathrm{~A}_{4}, \mathrm{~S}_{4}$, or $\mathbb{Z}_{7} \rtimes \mathbb{Z}_{3}$. Again by the Atlas [1], it is easily shown that all isomorphic subgroups of $V$ are conjugate in $\operatorname{Aut}(V)$. Thus we may assume that $K=L$, and so $\sigma$ is an automorphism of $K$. By [1], Aut $(K) \cong \mathrm{N}_{\mathrm{Aut}(V)}(K)<\operatorname{Aut}(V)$. Therefore, $\sigma$ extends to an automorphism of $V$, and so $\mathrm{L}_{2}(p)$ for $p \in\{5,7\}$ is homogeneous.

(2) Assume that $V=\mathrm{SL}_{2}(p)$, where $p=5$ or 7 .

Let $L$ be a subgroup of $V$. Then $L \cong L_{0}$ or $\mathbb{Z}_{2} \cdot L_{0}$, where $L_{0}$ is a subgroup of $\mathrm{L}_{2}(p)$. Since all isomorphic subgroups of $\mathrm{L}_{2}(p)$ are conjugate in $\operatorname{Aut}\left(\mathrm{L}_{2}(p)\right)$, it follows that all isomorphic subgroups of $V$ are conjugate in Aut $(V)$. Thus we only need to prove that each automorphism of $L$ extends to an automorphism of $V$. This is clearly true if $L \neq \mathbb{Z}_{4}, \mathbb{Z}_{8}$ as $\mathrm{L}_{2}(p)$ is homogeneous. Further, an element of $V$ of order 4 is conjugate to its inverse. Suppose that $L \cong \mathbb{Z}_{8}$. Then each element of $\operatorname{Aut}(L)$ is induced by an element of $\mathrm{N}_{\text {Aut }(V)}(L)$. Thus each element of $\operatorname{Aut}(L)$ extends to an automorphism of $V$. So $V$ is homogeneous.

This completes the proof of the theorem.

\section{REFERENCES}

[1] J.H. Conway, R.T. Curtis, S.P. Norton, R.A. Parker and R.A. Wilson, Atlas of finite groups (Clarendon Press, Oxford, 1985).

[2] G.L. Cherlin and U. Felgner, 'Quantifier eliminable groups', in Logic Colloquium 1980, (van Dalen, Editor) (North-Holland, Amsterdam, 1982), pp. 69-81.

[3] G.L. Cherlin and U. Felgner, 'Homogeneous solvable groups', J. London Math. Soc. (2) 44 (1991), 102-120.

[4] W. Feit and G.M. Seitz, 'On finite rational groups and related topics', Mlinois J. Math. 33 (1989), 103-131.

[5] C.H. Li, 'Isomorphisms of finite Cayley digraphs of bounded valency II', J. Combin. Theory Ser. $A$ (to appear). 
[6] C.H. Li and C.E. Praeger, 'The finite simple groups with at most two fusion classes of every order', Comm. Algebra 24 (1996), 3681-3704.

[7] C.H. Li and C.E. Praeger, 'Finite groups in which any two elements of the same order are either fused or inverse-fused', Comm. Algebra 25 (1996), 3081-3118.

[8] C.H. Li, C.E. Praeger and M.Y. Xu, 'Isomorphisms of finite Cayley digraphs of bounded valency', J. Combin. Theory Ser. B 73 (1998), 164-183.

[9] G. Stroth, 'Isomorphic subgroups', Comm. Algebra 24 (1996), 3049-3063.

[10] J.P. Zhang, 'On finite groups all of whose elements of the same order are conjugate in their automorphism groups', J. Algebra 153 (1992), 22-36.

Department of Mathematics

University of Western Australia

Perth. 6907 WA

Australia 